\title{
Strategies of engineering 2D nanomaterial-based electrocatalysts toward hydrogen evolution reaction
}

\author{
Yaping Chen ${ }^{1,2} \cdot$ Guoqiang Zhao ${ }^{1,2} \cdot$ Wenping Sun ${ }^{1}(\mathbb{C}$
}

Received: 28 February 2020 / Accepted: 27 April 2020 / Published online: 6 May 2020

(c) The Author(s) 2020

\begin{abstract}
Electrochemical water splitting driven by renewable energy-derived electricity is considered as the most promising pathway for delivering clean and sustainable hydrogen production. The key to achieving an efficient water splitting process is developing highly active electrocatalysts. Two-dimensional (2D) nanomaterials hold great promise in the electrocatalysis field due to their unique physicochemical properties. Some of them are not active enough because of the poor intrinsic activity, low density of active sites or low electrical conductivity. Some are inert for electrocatalytic reactions, but are able to work as the functional substrates for hybrid electrocatalysts. Thus, tremendous strategies have been developed to modulate the physicochemical and electronic properties of 2D nanomaterial-based electrocatalysts, and to make full use of the functionalities of functional 2D nanomaterial substrates to achieve fast catalytic reaction kinetics. In this review, the recent progress on the well-established design strategies for the 2D nanomaterials-based electrocatalysts is highlighted. The perspectives on the current challenges and future development of 2D electrocatalysts are addressed.
\end{abstract}

Keywords 2D nanomaterial $\cdot$ Electrocatalysts $\cdot$ Hydrogen evolution reaction $\cdot$ Electrochemical water splitting

\section{Introduction}

The energy shortage and environmental issues caused by the overuse of insufficient fossil fuels are becoming two urgent challenges for current society [1,2]. Exploiting the clean and renewable energy sources (e.g., wind, solar, etc.) is highly desirable to meet the long-term energy demand and environmental sustainability. However, the utilization efficiencies of renewable energies are always limited by their unpredictability and discontinuity. Thus, to make efficient use of renewable energy, developing advanced energy storage and conversion technologies is of great significance. Among the energy conversion technologies, one of the most attractive and promising options is renewable energy electricity-driven water splitting for hydrogen generation, which includes two

Wenping Sun

wenpingsun@zju.edu.cn

1 School of Materials Science and Engineering, State Key Laboratory of Silicon Materials, Zhejiang University, Hangzhou 310027, People's Republic of China

2 Institute for Superconducting and Electronic Materials, Australian Institute for Innovative Materials, University of Wollongong, Wollongong, NSW 2522, Australia electrochemical processes of hydrogen evolution reaction (HER) and oxygen evolution reaction (OER) [3, 4]. The key to the promotion of the water-splitting kinetics is to design highly active HER and OER electrocatalysts. To date, precious metal-based catalysts like $\mathrm{Ir}, \mathrm{Pt}$, and $\mathrm{RuO}_{2}$ are extensively employed for the two reactions. However, they suffer from high-cost, low-scarcity, and insufficient stability, which would severely limit their large-scale applications [5-10]. Therefore, seeking for cost-effective alternatives with high catalytic performance to noble metal-based electrocatalysts is greatly important for the development and substantial commercialization of economic water splitting technologies.

Since 2D graphene was discovered, numerous 2D nanomaterials have aroused plenty of research interest in the fields of energy conversion and storage [11, 12]. In particular, 2D nanomaterials have become one of the most promising candidate materials for the design and development of electrocatalysts due to their unique physic chemical properties and electronic structures [13-16]. However, some 2D nanomaterials are not active enough because of the poor intrinsic activity, low density of active sites or weak conductivity. Some 2D nanomaterials are inert for electrocatalysis, but can act as functional substrates to boost the catalytic activity of hybrid electrocatalysts. Thus, numerous strategies 
have been developed to modulate the physicochemical and electronic properties of 2D material-based electrocatalysts, and to make full use of the functionalities of functional 2D nanomaterial substrates to achieve fast catalytic reaction kinetics [17-23].

In this review, we summarized the design and construction strategies for 2D nanomaterial-based HER electrocatalysts. These strategies are categorized into five types, including heteroatom doping, defect engineering, interface engineering, phase engineering, and geometric engineering. The underlying mechanisms for enhancing the catalytic activity are also discussed. Finally, the challenges and perspectives for 2D material-based HER electrocatalysts are proposed.

\section{Engineering protocols for 2D electrocatalysts}

2D nanomaterials have been extensively applied in the electrocatalysts due to their adjustable and evenly exposed lattice planes, unique physicochemical properties, and electronic structures. These special features can have remarkable influences on the extrinsic and intrinsic activities of electrocatalysts. Apart from the adjustable catalytic activities, 2D nanomaterials can also work as functional supports because of their large specific surface areas and robustness for the development of hybrid electrocatalysts. However, most pure 2D electrocatalysts are inert or not active enough because of the poor intrinsic activity, low density of active sites, and low electrical conductivity [24-26]. Thus, some efficient optimization strategies (including heteroatom doping, surface/interface building, defect constructing, geometric controlling, etc.) are already proposed for 2D electrocatalysts to achieve higher catalytic activities. These optimization approaches aim to modulate the surface/interface properties and electronic structures correlated with hydrogen adsorption free energy and charge/mass transfer kinetics, which would affect catalytic kinetics. In this section, a variety of design strategies for 2D nanomaterial-based electrocatalysts are summarized and some typical demonstrations are highlighted.

\section{Heteroatom doping}

Heteroatom doping is considered as an efficient approach to modulating the electronic states, controlling the surface/ interface properties, and altering the elementary compositions of 2D electrocatalysts. So far, heteroatom doping method for 2D nanomaterials is mainly applied in the cases of layered double hydroxides (LDHs), graphene, transitionmetal dichalcogenides (TMDs), g- $\mathrm{C}_{3} \mathrm{~N}_{4}$, etc. Both non-metal atoms and metal atoms have been extensively studied as the dopants. Doping graphene with one or more non-metal heteroatoms is the most efficient way for boosting electrocatalytic performance of graphene-based catalysts [27]. Among the single-heteroatom-doped graphene nanomaterials, $\mathrm{N}$-doped graphene-based catalysts are the pioneer of nonmetal-atom doped 2D electrocatalysts. $\mathrm{N}$ atom is the most common and efficient dopant for carbonaceous materials. Since the size and electronegativity of $\mathrm{N}$ atoms are different from those of $\mathrm{C}$ atoms, the electronic states of carbonaceous catalysts could be modulated by $\mathrm{N}$ doping via the conjugation effects between nitrogen lone electronic pairs and carbon $\pi$-system [28, 29]. B-doped graphene exhibits exceptional HER performance [30], but the activity is still inferior to those of the $\mathrm{MoS}_{2}$-based and Pt-based electrocatalysts. Moreover, DFT calculations found that although doping heteroatoms in graphene can enhance the hydrogen adsorption ability, all the $\Delta G_{\mathrm{H}^{*}}$ values are still positive, demonstrating unfavorable thermodynamic adsorption process. Thus, all heteroatom-doped graphene catalysts locate at the right branch bottom in the volcano curves (Fig. 1a) [30]. To rank the catalytic activity of graphene-based electrocatalysts in volcano plots, a basic principle of optimizing $\Delta G_{\mathrm{H}^{*}}$ was proposed by Qiao et al. according to the Hückel molecular orbital theory (HMOT). The results showed that the higher the density of state (DOS) position, the better the linearity between the DOS and the $\Delta G_{\mathrm{H}^{*}}$, which also indicates the stronger hydrogen adsorption strength (Fig. 1b). In addition, they demonstrated that the position of the DOS closer to the Fermi level shows stronger interaction with the adsorbed hydrogen intermediates, accordingly achieving a lower $\Delta G_{\mathrm{H}^{*}}$ value. Motivated by the findings, $\mathrm{N}, \mathrm{P}$, $\mathrm{S}, \mathrm{Br}$, and $\mathrm{O}$ heteroatoms were investigated as the dopants. These heteroatoms commonly substitute or disrupt the carbon atoms at the edge areas and basal planes to form more defect sites, which can work as the active sites, facilitating the eletrocatalytic processes [31,32]. As is well known, the catalytic activity is dominated by the density of the exposed active sites and the intrinsic activity of active centers that are correlated with the extrinsic geometric characteristics. For the doped graphene-based catalysts, the total exposure number of active sites is closely connected with the doping levels and specific surface area. Thus, in addition to the selection of doped heteroatoms, the doping degree and specific surface area should also be taken into account for enhancing HER activities of graphene-based catalysts.

In addition to heteroatom-doped 2D carbon-based electrocatalysts as discussed in the previous sections, doping 2D TMD-based electrocatalysts with heteroatoms can also modulate the catalytic activities. Heteroatom doping can efficiently create additional active sites and tune the electronic states of TMDs, directly enhancing the extrinsic and intrinsic activity [33-35]. Our group investigated $\mathrm{Ni} /$ Co-doped $\mathrm{MoSe}_{2}$ nanosheets as an effective alkaline HER 

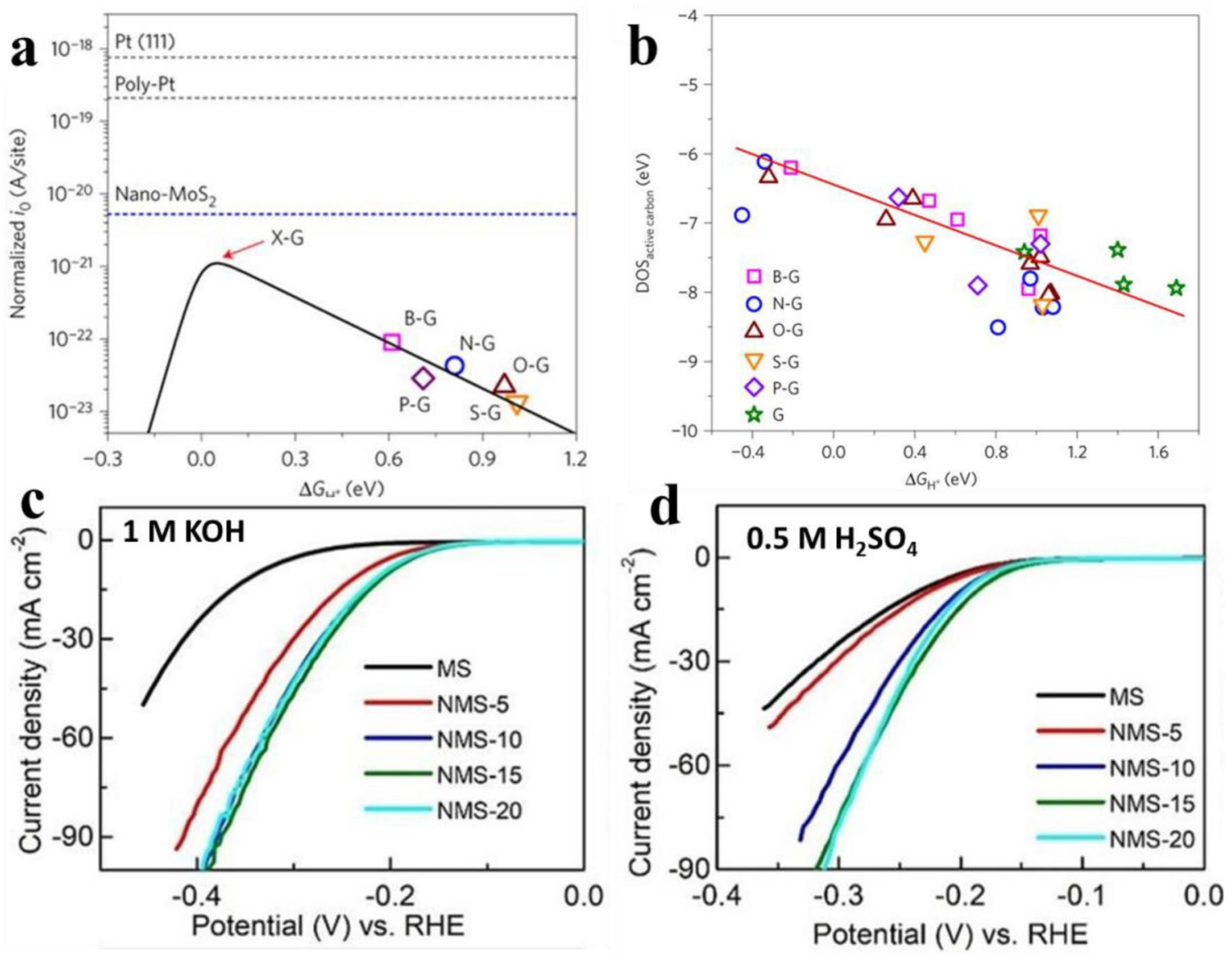

Fig. 1 a HER volcano plots containing $i_{0 / \text { site }}{ }^{\text {theory }}$ (solid black curve) and $i_{0 / \text { site }}$ experiment obtained from Tafel plots (colored open symbols) versus $\Delta G_{\mathrm{H}}^{*}$ for each graphene model. The blue and gray dashed lines from nano- $\mathrm{MoS}_{2}$ and single-crystal and polycrystalline $\mathrm{Pt}$ as the targets for optimization. b The relationship between $\Delta G_{\mathrm{H}}^{*}$ and the highest

peak position of DOS of the graphene-based materials; the red line is a guide for the eye [30], $\mathbf{c}, \mathbf{d ~ L S V}$ curves of the $\mathrm{MoSe}_{2}$ and $\mathrm{Ni}$-doped $\mathrm{MoSe}_{2}$ catalysts in alkaline and acidic media, respectively [36]

electrocatalysts [36]. Ni- or Co-doped $\mathrm{MoSe}_{2}$ catalysts deliver a slight promotion of acidic HER performance, but a substantial enhancement of HER activity in alkaline media as compared with bare $\mathrm{MoSe}_{2}$ (Fig. 1c, d). Experimental results and DFT calculations demonstrate that Ni or Co doping, in addition to boosting the ability of water adsorption, optimizes the hydrogen adsorption free energy $\left(\Delta G_{\mathrm{H}}\right)$ on the basal plane of $\mathrm{MoSe}_{2}$ nanosheets. Besides, doping $\mathrm{Fe}, \mathrm{Co}$, or $\mathrm{Ni}$ etc. could also activate inert basal planes, such as the activation of in-plane $\mathrm{S}$ sites, which can greatly increase the amount of active sites [37-39].

\section{Defect engineering}

Engineering defects (nano nano-holes, vacancies, etc.) is an efficient way to modulate the electronic structures and physicochemical properties of 2D nanomaterial-based electrocatalysts, which can strongly affect their extrinsic and intrinsic activities [40-43]. Some reports claimed that the

catalytic activity of $\mathrm{MoS}_{2}$ originates from the edge Mo sites $[44,45]$. Introducing $\mathrm{S}$ vacancies into the basal planes can not only modify the band gaps but also create additional exposed Mo edge sites [44, 46, 47]. Xie's group utilized a large-scale approach to introducing defects on the surface of $\mathrm{MoS}_{2}$ to generate more active edges [48]. The HRTEM results confirm the formation of disordered atomic arrays on the basal planes, which can cause the crack of the basal planes, generating more additional edges (Fig. 2a-c). Ajayan's group constructed abundant defects within the monolayer of $\mathrm{MoS}_{2}$ via hydrogen treatment and oxygen plasma exposure [49]. The formation of these defects significantly increases the total number of the exposed active edge sites, significantly enhancing HER kinetics (Fig. 2d-g). Moreover, the constructed defects can cause the lattice strains, which also modulate the band gaps of $2 \mathrm{D}$ materials. $\mathrm{Li}$ et al. introduced $\mathrm{S}$ vacancies and strains in the monolayer $2 \mathrm{H}-\mathrm{MoS}_{2}$ basal plane for improved HER activity [50]. The S vacancies with band states approaching the Fermi level can

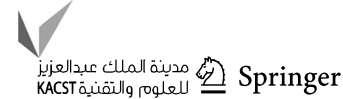



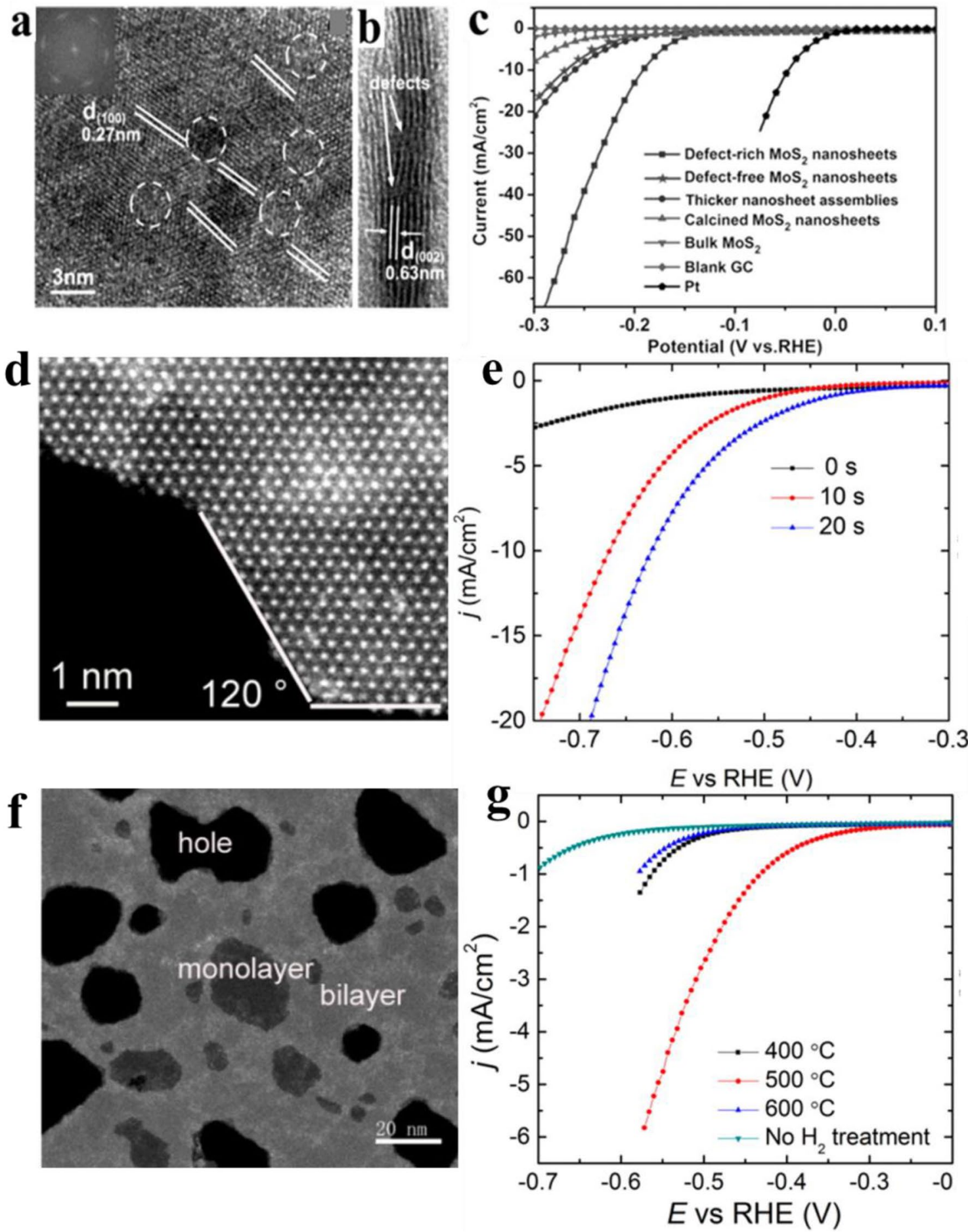

Fig. 2 a HRTEM image and the corresponding FFT patterns of the defect-rich $\mathrm{MoS}_{2}$ ultrathin nanosheets. b Cross-sectional HRTEM image of the defect-rich $\mathrm{MoS}_{2}$ ultrathin nanosheets. c Polarization curves of the defect-free $\mathrm{MoS}_{2}$ nanosheets, defect-rich $\mathrm{MoS}_{2}$ nanosheets, thicker $\mathrm{MoS}_{2}$ nanosheet assemblies, calcined $\mathrm{MoS}_{2}$ nanosheets, bulk $\mathrm{MoS}_{2}$, blank glassy carbon, and Pt [44], d STEM

images of Mo and $\mathrm{S}_{2}$ terminated edges created by the oxygen plasma in $\mathrm{MoS}_{2}$ with $120^{\circ}$ angle. e The LSV curves of $\mathrm{MoS}_{2}$ before and after treated by oxygen plasma. $\mathbf{f}$ TEM images of the exposed edges and step-edges of the $\mathrm{MoS}_{2}$ layers. g Polarization curves of $\mathrm{MoS}_{2}$ with hydrogen annealing at various temperatures [45] 
facilitate hydrogen adsorption. The $\Delta G_{\mathrm{H}}$ can be dominated by strains induced by $\mathrm{S}$ vacancies, and thereby improving the HER kinetics. The synergistic effects of $\mathrm{S}$ vacancies and strains lead to an optimal $\Delta G_{\mathrm{H}}=0 \mathrm{eV}$ and attain significantly enhanced intrinsic HER activity. Thus, constructing defects for 2D nanomaterial-based electrocatalysts has been an important strategy for accelerating catalytic activity. However, controllable tuning of defects is necessary for the in-depth understanding of defects and the effects on reaction mechanisms. For example, Yao's group engineered defectabundant graphene via removing the doped $\mathrm{N}$ [51]. They found that the promoted catalytic performance is strongly correlated with the defect contents induced by the removal of nitrogen, and the lower the nitrogen content, the higher the catalytic performance, further indicating that defectengineering strategy is feasible to achieve high catalytic activity for graphene-based catalysts.

\section{Interface engineering}

Interface engineering is an essential strategy to constructing highly active catalysts with well-defined heterointerfaces. Engineering hetero-interface employing different components can modulate the surface/interface properties and electronic structures of 2D nanomaterial-based electrocatalysts, inducing novel physicochemical properties and synergistic effects, which are beneficial to the enhancement of electrocatalytic activities [23, 52]. Our group reported $\mathrm{Ni}(\mathrm{OH})_{2} /$ $\mathrm{MoS}_{2}$ heterostructures with $\mathrm{Ni}(\mathrm{OH})_{2}$ nanoclusters decorated on $\mathrm{MoS}_{2}$ nanosheets for improved alkaline HER performance (Fig. 3a-c) [53]. The $\mathrm{Ni}(\mathrm{OH})_{2}$ nanoclusters can not only improve the water adsorption/dissociation process, but also tune the electronic structures of $\mathrm{MoS}_{2}$, thereby synergistically enhancing alkaline HER kinetics. After that, our group further proposed to decorate the basal planes of molybdenum dichalcogenides with $\mathrm{SnS}_{2}$ quantum dots for alkaline HER (Fig. 3d) [54]. The optimal $\mathrm{MoSe}_{2} / \mathrm{SnS}_{2}$ heterostructured catalyst delivers significantly enhanced alkaline HER activity over pure $\mathrm{MoSe}_{2}$ in 1-M KOH (Fig. 3e). The alkaline HER activity enhancement is mainly ascribed to the accelerated rate-determining water adsorption/dissociation kinetics. Meanwhile, the DFT calculations revealed that $\mathrm{SnS}_{2}$ highly boosts the water adsorption capability of $\mathrm{MoSe}_{2}$ nanosheets, which is critical for the following water dissociation process (Fig. 3f). Similarly, $\mathrm{MoS}_{2} / \mathrm{MoSe}_{2}$ heterostructures with $\mathrm{MoS}_{2}$ nanoclusters anchored on $\mathrm{MoSe}_{2}$ nanosheets for enhanced alkaline HER were also studied by our group [55]. $\mathrm{MoS}_{2}$ nanoclusters were anchored on the $\mathrm{MoSe}_{2}$ nanosheets, leading to more additional edge active sites (Fig. 3g-h). The optimal $\mathrm{MoS}_{2} / \mathrm{MoSe}_{2}$ heterostructured electrocatalyst delivers a substantially accelerated catalytic activity in 1-M KOH as compared with the bare $\mathrm{MoSe}_{2}$ and $\mathrm{MoS}_{2}$ nanosheets (Fig. 3i).
Apart from 2D TMD-based heterostructures, metalfree 2D materials can be also employed to construct heterostructures. Qiao's group coupled g- $\mathrm{C}_{3} \mathrm{~N}_{4}$ with $\mathrm{N}$-doped graphene $\left(\mathrm{C}_{3} \mathrm{~N}_{4} @ \mathrm{NG}\right)$ as a heterostructured catalyst for enhanced HER kinetics [25]. Combining the experimental findings and the DFT calculations, they found that the promoted HER activities originate from the synergistic effects of heterostructures. The $\mathrm{g}-\mathrm{C}_{3} \mathrm{~N}_{4}$ contributes to the hydrogen adsorption and $\mathrm{N}$-doped graphene enhances the electrontransfer ability (Fig. 4a,b). Later, they fabricated $\mathrm{N}$-doped graphene nanosheets with porous $\mathrm{C}_{3} \mathrm{~N}_{4}$ nanolayers as the free-standing HER electrocatalyst [56]. The heterostructured electrocatalyst achieves exceptional HER performance with larger exchange current density, lower onset potential, and better durability as compared to commercial Pt/C. The outstanding HER performance originates from the synergistic effect of heterostructures, where in-plane porous $\mathrm{C}_{3} \mathrm{~N}_{4}$ provides the exposed active sites and $3 \mathrm{D}$ conductive graphene network offers more electron channels. To sum up, the heterostructure design principles for 2D nanomaterialbased electrocatalysts are commonly based on the following several factors. Firstly, to fabricate the heterostructures, two (more) dissimilar components are required. Secondly, at least one component possesses catalytic activity. Thirdly, the different components should play a complementary role in electrocatalytic process. Finally, it would be better if the heterostructured catalysts are highly conductive for fast electron transfer.

Besides, atomically dispersing metal atoms on the 2D substrates are also recognized as a promising pathway to regulating the catalytic activity, because the well-defined atomic interface between single metal sites and 2D platforms can achieve the precise modulation of the local coordination environment and electronic structures, which are closely correlated with the catalytic kinetics [57]. For example, Qi et al. reported single cobalt atoms bonded to the distorted $1 \mathrm{~T} \mathrm{MoS}_{2}$ nanosheets (SA Co-D 1T $\mathrm{MoS}_{2}$ ) as an efficient HER electrocatalyst, delivering high activity and long-term stability [58]. During the synthesis process of the SA Co-D $1 \mathrm{~T} \mathrm{MoS}_{2}$ catalyst, the formation of $\mathrm{Co}-\mathrm{S}$ bond and lattice mismatch at the interface between $\mathrm{MoS}_{2}$ and Co generate strains, which could induce the phase transition from $2 \mathrm{H} \mathrm{MoS}_{2}$ to distorted 1T phase. DFT calculations demonstrate that the extraordinary HER activity is attributed to the ensemble effect at the interface between the Co single atoms and the D-1T MoS ${ }_{2}$ substrates, which can optimize the hydrogen binding energy. Zhang et al. prepared single atom Pt on the MXene support for the enhanced HER activity [59]. The modified $\mathrm{Mo}_{2} \mathrm{TiC}_{2} \mathrm{~T}_{\mathrm{x}}$ nanosheets with abundant Mo vacancies provide large amount of anchoring sites for immobilizing $\mathrm{Pt}$ active centers to form $\mathrm{Pt}-\mathrm{C}$ and $\mathrm{Pt}-\mathrm{O}$ atomic interfaces. The developed catalyst shows an outstanding HER activity and high durability. DFT calculations 

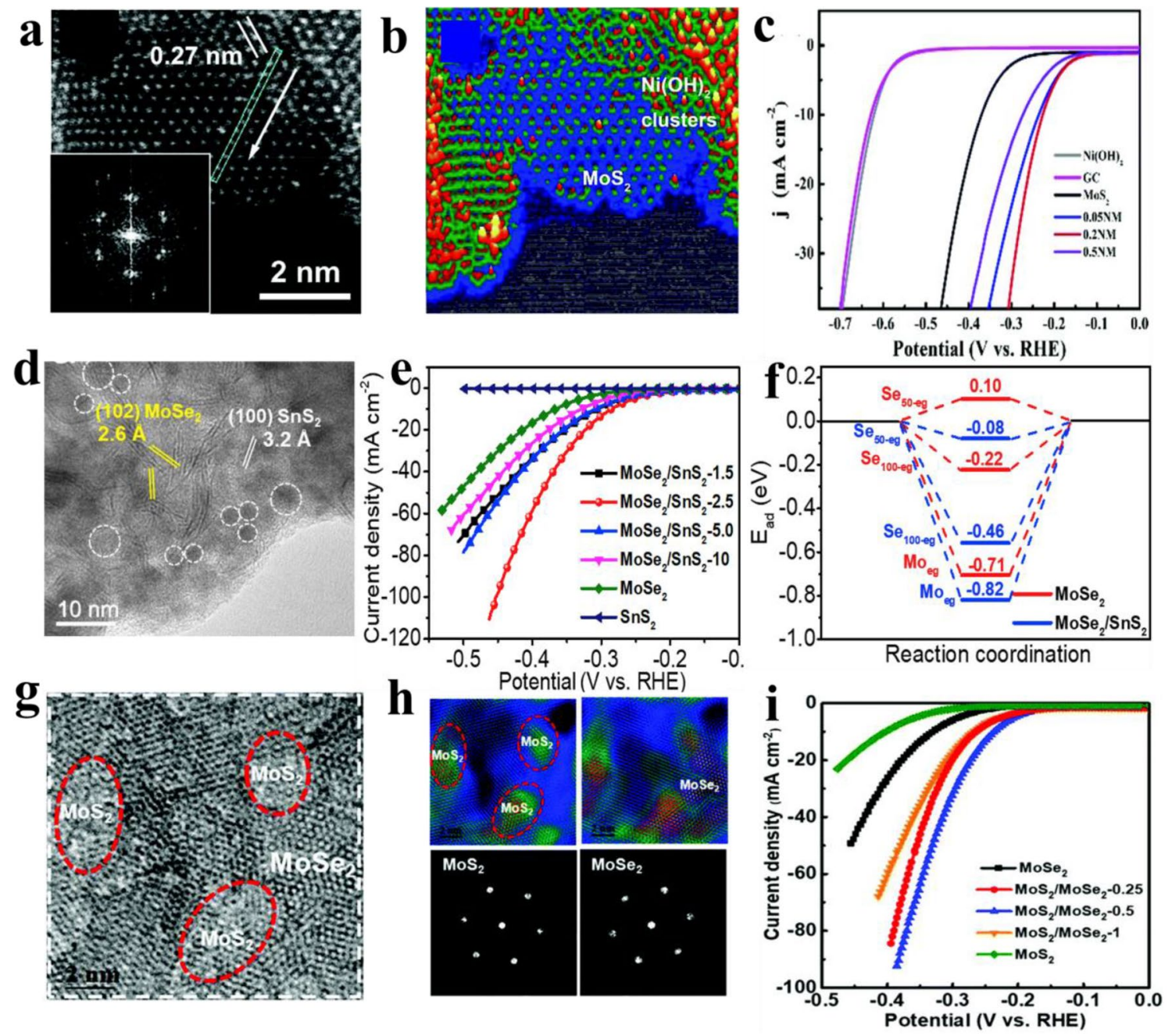

Fig. 3 a HAADF-STEM image of $\mathrm{Ni}(\mathrm{OH})_{2} / \mathrm{MoS}_{2}$ heterostructures. Insets: the corresponding FFT patterns. $\mathbf{b}$ The surface plot image of (a). c LSV curves of the $\mathrm{Ni}(\mathrm{OH})_{2}, \mathrm{MoS}_{2}$, and $0.05 \mathrm{NM}, 0.2 \mathrm{NM}$, and $0.5 \mathrm{NM}$ (NM: the abbreviation of $\mathrm{Ni}(\mathrm{OH})_{2} / \mathrm{MoS}_{2}$ heterostructure) [49], d TEM images of $\mathrm{MoSe}_{2} / \mathrm{SnS}_{2}-2.5$ heterostructure. e Polarization curves of $\mathrm{MoSe}_{2} / \mathrm{SnS}_{2}$ heterostructures, $\mathrm{SnS}_{2}$, and $\mathrm{MoSe}_{2}$. f

reveal that the $\mathrm{Mo}_{2} \mathrm{TiC}_{2} \mathrm{O}_{2}-\mathrm{Pt}_{\mathrm{SA}}$ shows higher electronic energy levels and enhanced ability of electron donor than bare $\mathrm{Mo}_{2} \mathrm{TiC}_{2} \mathrm{O}_{2}$, and achieves an optimal hydrogen adsorption free energy. Cheng et al. synthesized single Pt atoms dispersed on aniline-stacked graphene (Pt SASs/AG) via a microwave reduction method for the enhanced HER kinetics (Fig. 4c) [60]. The Pt SASs/AG catalyst delivers an outstanding catalytic performance with overpotential of $12 \mathrm{mV}$ at $10 \mathrm{~mA} \mathrm{~cm}{ }^{-2}$ (Fig. 4d). Also, the mass activity is 46 times higher than that of commercial $20 \mathrm{wt} \% \mathrm{Pt} / \mathrm{C}$. Besides, the Pt SASs/AG sample achieves a remarkable stability as compared with Pt/C. X-ray absorption fine structure (XAFS) and DFT calculations demonstrate that the coordination of the $\mathrm{N}$ atoms originating from aniline can effectively modulate the electronic structures of Pt atoms and optimize the hydrogen
Water adsorption energy diagram for $\mathrm{MoSe}_{2} / \mathrm{SnS}_{2}$ heterostructures and pure $\mathrm{MoSe}_{2}$ [50], g HRTEM image of $\mathrm{MoS}_{2} / \mathrm{MoSe}_{2}$ heterostructure. $\mathbf{h}$ IFFT image from the selected masked FFT patterns (top) and the selected FFT patterns (down) for $\mathrm{MoSe}_{2}$ and $\mathrm{MoS}_{2}$. i LSV curves of $\mathrm{MoS}_{2} / \mathrm{MoSe}_{2}$ heterostructures, $\mathrm{MoS}_{2}$, and $\mathrm{MoSe}_{2}$ in $1-\mathrm{M} \mathrm{KOH}$ solution [51]

adsorption energy, thereby boosting the HER kinetics. Apart from noble-metal single atoms, a number of transition-metal single atoms (e.g., Co and $\mathrm{Ni}$ ) anchored on the functionalized graphene supports as HER electrocatalysts were also reported, and delivered promising catalytic performance $[61,62]$.

\section{Phase engineering}

Phase engineering for TMDs (from the semiconducting $2 \mathrm{H}$ phase to the metastable metallic $1 \mathrm{~T}$ phase) can alter the bandgaps and enhance electronic conductivity. Generally, the strategies for phase engineering include alkali metal intercalation [63, 64], hydrothermal method [65], heterostructure construction [66], etc. Chhowalla's group utilized 
Fig. 4 a The polarization plots for g- $_{3} \mathrm{~N}_{4}, \mathrm{~N}$-graphene, $\mathrm{C}_{3} \mathrm{~N}_{4} /$ $\mathrm{NG}$ mixture, $\mathrm{C}_{3} \mathrm{~N}_{4} @ \mathrm{NG}$, and $20 \% \mathrm{Pt} / \mathrm{C}$ in $0.5 \mathrm{M} \mathrm{H}_{2} \mathrm{SO}_{4}$ at the scan rate of $5 \mathrm{mV} \mathrm{s}^{-1}$. $\mathbf{b}$ The free-energy diagram calculated at the equilibrium potential for $\mathrm{N}$-graphene, $\mathrm{C}_{3} \mathrm{~N}_{4} / \mathrm{NG}$ mixture, $\mathrm{C}_{3} \mathrm{~N}_{4} @ \mathrm{NG}$, and $\mathrm{Pt}$ [25], c HAADF-STEM images of Pt SASs/AG. d LSV curves of $\mathrm{Pt} \mathrm{SASs} / \mathrm{AG}$ and $\mathrm{Pt} / \mathrm{C}$ in $0.5-\mathrm{M} \mathrm{H}_{2} \mathrm{SO}_{4}$ at the scan rate of $2 \mathrm{mV} \mathrm{s}^{-1}$ [56]
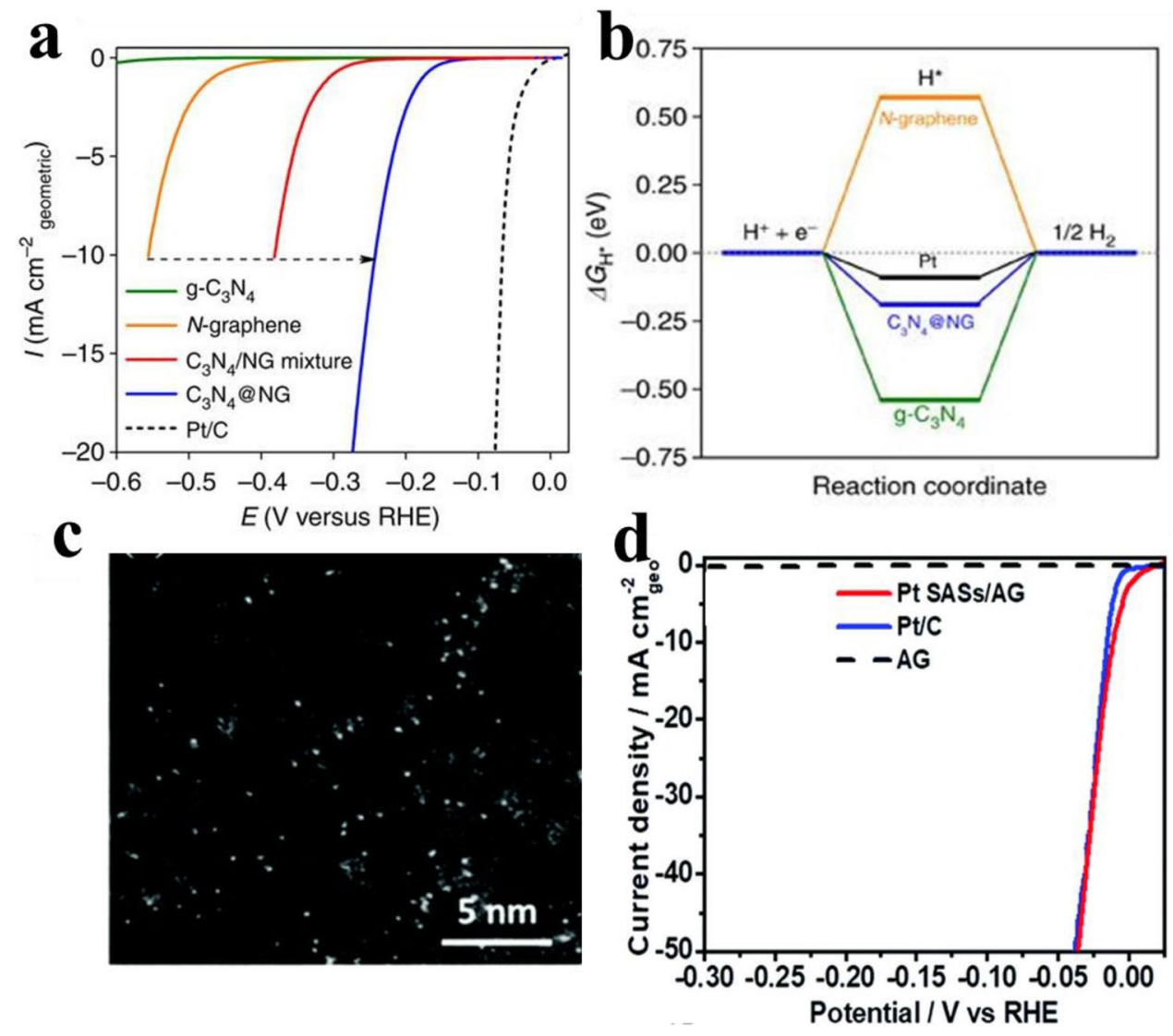

the solvent-free intercalation method to obtain the exfoliated $\mathrm{MoS}_{2}$ nanosheets with a large amount of metallic 1T phases as the highly active HER electrocatalysts [63]. The $\mathrm{MoS}_{2}$ nanosheets of $1 \mathrm{~T}$ phase deliver superior HER kinetics after the removal of surface excess negative charges. Moreover, the HER performance of $2 \mathrm{H}$ phase can be significantly promoted by adding the conductive SWNTs, indicating that the poor conductivity of $2 \mathrm{H}$ phase is unfavorable to the catalytic activity. The results further demonstrate that $1 \mathrm{~T}$ phase shows a better electron-transfer property than $2 \mathrm{H}$ phase, which is an important factor for the enhancement of the HER performance [63]. Similarly, their group exfoliated $\mathrm{WS}_{2}$ nanosheets with a large amount of metallic $1 \mathrm{~T}$ phase via lithium intercalation way as an efficient HER electrocatalysts [67]. The HADDF images confirm the presence of the zigzag structure in metallic $1 \mathrm{~T}$ phase, which can induce bond strain, greatly improving the HER kinetics (Fig. 5a). Besides, the exfoliated $1 \mathrm{~T} \mathrm{WS}_{2}$ shows excellent durability (Fig. 5b). DFT calculations further demonstrate that tensile strain is beneficial to enhance catalytic activity for $\mathrm{WS}_{2}$ nanosheets. Therefore, the phase transition of TMDs from semiconductor $2 \mathrm{H}$ phase to metallic $1 \mathrm{~T}$ phase via chemical exfoliation is an efficient way for the promotion of HER catalytic activity. Besides, Jin's group prepared partially crystallized $1 \mathrm{~T}-\mathrm{MoSe}_{2}$ nanosheets composed of crystalline and disordered phase via a hydrothermal strategy [68]. They claimed that the $1 \mathrm{~T}$ phase can effectively enhance the intrinsic activity and conductivity of the electrocatalysts; while, the disordered phase can offer plentiful defective sites as the active centers. This synergistic effect results in superior activity with overpotential of $152 \mathrm{mV}$ at $10 \mathrm{~mA} \mathrm{~cm}^{-2}$ (Fig. 5c). 1T phase of TMDs is very unstable and tends to convert into thermodynamically stable $2 \mathrm{H}$ phase. Electron injection from additional components would be an efficient way to stabilize $1 \mathrm{~T}$ TMDs phase. Zhang et al. reported $1 \mathrm{~T}-\mathrm{MoSe}_{2} / \mathrm{NiSe}$ heterostructures with $1 \mathrm{~T}-\mathrm{MoSe}_{2}$ nanosheets anchored on metallic NiSe nanowires [66]. The NiSe nanowires could inject electrons into $\mathrm{MoSe}_{2}$ nanosheets, leading to the transition of $\mathrm{MoSe}_{2}$ from $2 \mathrm{H}$ phase to $1 \mathrm{~T}$ phase (Fig. 5d-f). Benefitting from the phase transition, the hybrid electrocatalysts display excellent HER activity and decent durability. This strategy can be also applied to designing other 1T $\mathrm{MX}_{2}$-based heterostructures for various application fields.

\section{Geometric engineering}

The geometric configurations of 2D electrocatalysts significantly affect the catalytic performance due to the following reasons [69]: (1) decreasing the lateral size of 2D 

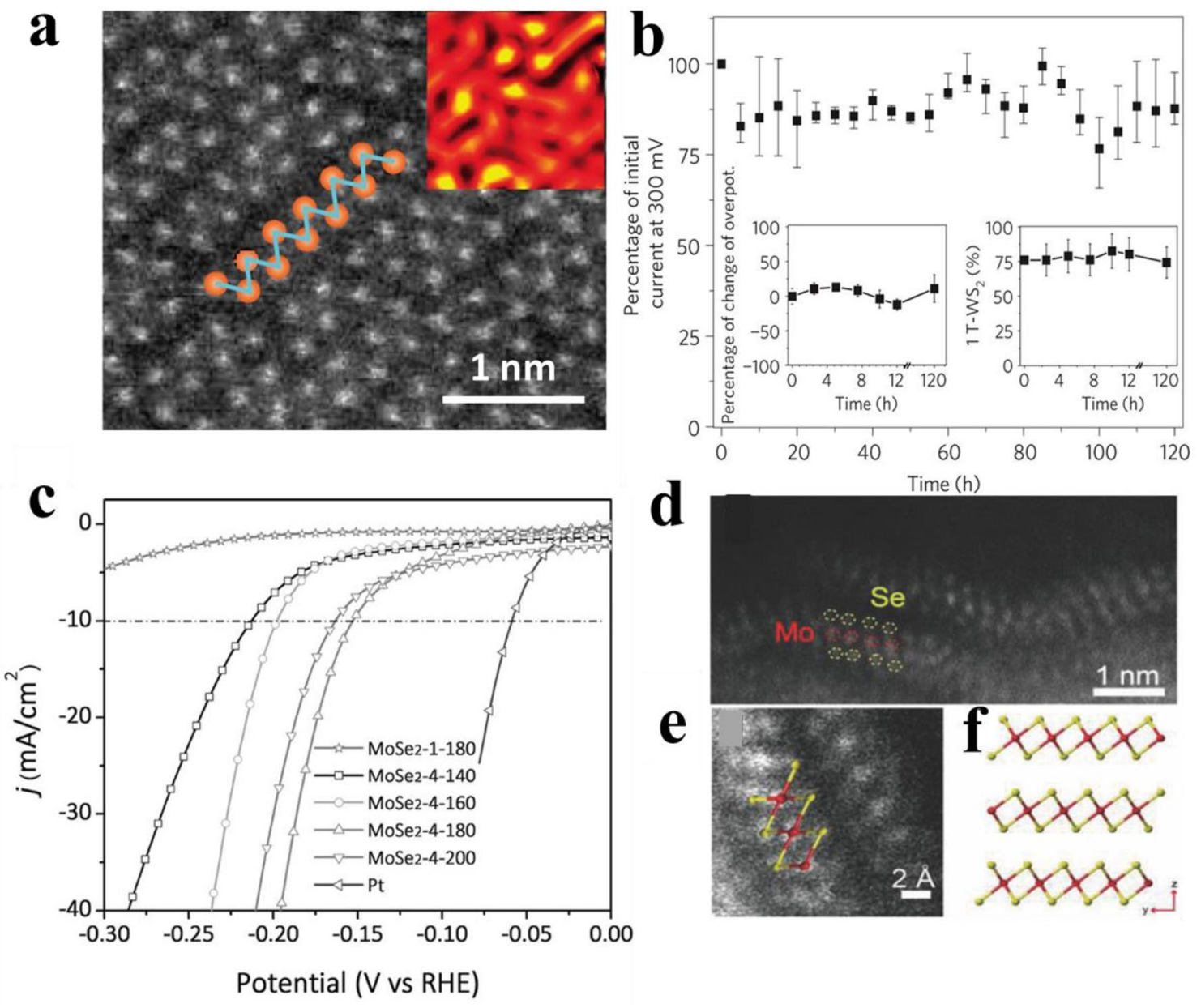

Fig. 5 a STEM image for the exfoliated $\mathrm{WS}_{2}$ monolayer showing $1 \mathrm{~T}$ superlattice. b The variation in current density as a function of time for $1 \mathrm{~T} \mathrm{WS}_{2}$ electrode. The insets: the percentage of change in overpotential (left) and variation in $1 \mathrm{~T}$ phase concentration versus time

electrocatalysts could expose more active sites [70]; (2) altering the thickness of 2D nanomaterials could modulate the electronic properties [71]; (3) reducing the thickness of 2D nanomaterials to the atomic scale is beneficial to generating more in-plane defects as additional active sites, and optimizing the electrical conductivity [72]; Li et al. reported that the ultrasmall $\mathrm{MoS}_{2}$ particles with enrichment of $\mathrm{S}$ edges delivered better HER activity than the bulk counterpart [73]. Chhowalla's group discovered that reducing the thickness of $\mathrm{WS}_{2}$ to monolayer can not only increase the exposed active sites but also induce the lattice distortions, which lead to abundant defects for hydrogen evolution [67]. Cao's group found that the HER activity of $\mathrm{MoS}_{2}$ could be decreased by a factor of $\sim 4.47$ with increasing one additional atomic layer [74]. In comparison with the traditional views, they argued that increasing the atomic layer would hinder the hopping of electrons in the vertical direction of $\mathrm{MoS}_{2}$ layers. (right) [63], $\mathbf{c}$ Polarization curves of various $\mathrm{MoSe}_{2}$ and Pt samples [64], d, e $\mathrm{MoSe}_{2}$ nanosheet shell showing 1T-phase structure, f Crystal structure model of $1 \mathrm{~T}-\mathrm{MoSe}_{2}$ [62]

\section{Conclusions and outlook}

In this review, we summarized the design strategies of $2 \mathrm{D}$ nanomaterial-based HER electrocatalysts, including heteroatom doping, defect engineering, interface engineering, phase engineering, and geometric engineering. These strategies can efficiently optimize the electronic states, surface/ interface properties, electrical conductivity, and exposed active sites of electrode materials. Even though extensive efforts have been devoted to the design of 2D nanomaterialbased electrocatalysts, it remains challenging to deliver precisely controlled synthesis, determination of real active sites, understanding of the relevant mechanisms, and cost-effective commercial applications.

First, one of the greatest challenges is how to precisely control the surface and interface chemistry in 2D nanomaterials-based electrocatalysts at atomic scale with 
desired composition, defect structure and concentration, facet orientation, coordination structure, and so forth. Second, ex situ characterizations are generally applied in the investigation of the electrocatalysts and electrocatalytic reactions. The structure evolution and surface chemistry of the electrocatalysts during the electrocatalytic reactions cannot be well illustrated, which are also the typical issues in the electrocatalysis field. Furthermore, it is still very challenging to monitor the adsorption/desorption behaviors of the reaction intermediates, and, hence, one cannot capture a clear picture of the reaction processes and reaction mechanisms as well. Thus, advanced in situ/operando characterization techniques and protocols should be extensively developed to disclose the underlined structure-property relationship of the electrocatalysts and the relevant mechanisms. Third, in addition to the typical 2D TMDbased and the graphene-based electrocatalysts, some other types of 2D nanomaterials also have great potential for the development of advanced heterostructured/hybrid HER electrocatalysts, especially alkaline HER electrocatalysts. 2D-layered metal hydroxides like $\mathrm{Ni}(\mathrm{OH})_{2}$ and $\mathrm{Co}(\mathrm{OH})_{2}$ can serve as multifunctional substrates for constructing heterostructured alkaline HER electrocatalysts. They could not only serve as the supports for active species but also work as the co-catalysts for accelerating the rate-determining step of alkaline HER. With regard to alkaline HER, indepth understanding of the reaction mechanism remains to be the research frontier. The $\mathrm{g}-\mathrm{C}_{3} \mathrm{~N}_{4}$ and h-BN nanosheets, which are highly stable and contain large amount of $\mathrm{N}$, are also promising functional substrates for engineering heterostructured electrocatalysts. However, they are semiconductor or insulator, and possess insufficient electrical conductivity and low electrocatalytic activity. 2D metal carbides/nitrides possess high intrinsic activity [75, 76], but the lack of facile synthesis techniques would hamper their applications. Therefore, substantial efforts should be made to develop proper strategies to make full use of the advantages of these 2D nanomaterials towards the development of efficient HER electrocatalysts. Finally, there is a long way to go for 2D nanomaterial-based electrocatalysts towards practical applications. To date, there is lack of detailed performance evaluation in prototype water electrolyzers based on polymer electrolyte membranes. And, the electrocatalyst production is still at lab scale, and intensive research on large-scale production processes is urgently required.

Acknowledgements W. P. Sun acknowledges funding support from "Hundred Talents Program" of Zhejiang University, China and the Fundamental Research Funds for the Central Universities, China. This work was partially supported by an Australian Research Council (ARC) DECRA Grant (DE160100596) and a Discovery Project
(DP200100365). Y. P. Chen thank the funding support from the China Scholarship Council (CSC).

Open Access This article is licensed under a Creative Commons Attribution 4.0 International License, which permits use, sharing, adaptation, distribution and reproduction in any medium or format, as long as you give appropriate credit to the original author(s) and the source, provide a link to the Creative Commons licence, and indicate if changes were made. The images or other third party material in this article are included in the article's Creative Commons licence, unless indicated otherwise in a credit line to the material. If material is not included in the article's Creative Commons licence and your intended use is not permitted by statutory regulation or exceeds the permitted use, you will need to obtain permission directly from the copyright holder. To view a copy of this licence, visit http://creativecommons.org/licenses/by/4.0/.

\section{References}

1. Shi, Y., Zhang, B.: Recent advances in transition metal phosphide nanomaterials: synthesis and applications in hydrogen evolution reaction. Chem. Soc. Rev. 45, 1529-1541 (2016)

2. Wu, K.-H., Zeng, Q., Zhang, B., Leng, X., Su, D.-S., Gentle, I.R., Wang, D.-W.: Structural origin of the activity in Mn3O4-graphene oxide hybrid electrocatalysts for the oxygen reduction reaction. ChemSusChem. 8, 3331-3339 (2015)

3. Chen, Y., Rui, K., Zhu, J., Dou, S.X., Sun, W.: Recent progress on nickel-based oxide/(Oxy)hydroxide electrocatalysts for the oxygen evolution reaction. Chem. Eur. J. 25, 703-713 (2019)

4. Xie, Y., Cai, J., Wu, Y., Zang, Y., Zheng, X., Ye, J., Cui, P., Niu, S., Liu, Y., Zhu, J., Liu, X., Wang, G., Qian, Y.: Boosting water dissociation kinetics on $\mathrm{Pt}-\mathrm{Ni}$ nanowires by $\mathrm{N}$-induced orbital tuning. Adv. Mater. 31, 1807780 (2019)

5. Zhao, G., Rui, K., Dou, S.X., Sun, W.: Heterostructures for electrochemical hydrogen evolution reaction: a review. Adv. Funct. Mater. 28, 1803291 (2018)

6. Zheng, X., Chen, Y., Zheng, X., Zhao, G., Rui, K., Li, P., Xu, X., Cheng, Z., Dou, S.X., Sun, W.: Electronic structure engineering of $\mathrm{LiCoO} 2$ toward enhanced oxygen electrocatalysis. Adv. Energy Mater. 9, 1803482 (2019)

7. Zang, Y., Niu, S., Wu, Y., Zheng, X., Cai, J., Ye, J., Xie, Y., Liu, Y., Zhou, J., Zhu, J., Liu, X., Wang, G., Qian, Y.: Tuning orbital orientation endows molybdenum disulfide with exceptional alkaline hydrogen evolution capability. Nat. Commun. 10, 1217 (2019)

8. Li, C., Baek, J.-B.: Recent advances in noble metal (Pt, Ru, and Ir)-based electrocatalysts for efficient hydrogen evolution reaction. ACS Omega (2019). https://doi.org/10.1021/acsomega.9b03550

9. Bae, S.-Y., Mahmood, J., Jeon, I.-Y., Baek, J.-B.: Recent advances in ruthenium-based electrocatalysts for the hydrogen evolution reaction. Nanosc. Horizons. 5, 43-56 (2020)

10. Zhang, J., Liu, J., Xi, L., Yu, Y., Chen, N., Sun, S., Wang, W., Lange, K.M., Zhang, B.: Single-atom Au/NiFe layered double hydroxide electrocatalyst: probing the origin of activity for oxygen evolution reaction. J. Am. Chem. Soc. 140, 3876-3879 (2018)

11. Yang, Z., Ren, J., Zhang, Z., Chen, X., Guan, G., Qiu, L., Zhang, Y., Peng, H.: Recent advancement of nanostructured carbon for energy applications. Chem. Rev. 115, 5159-5223 (2015)

12. Zhang, H.: Ultrathin two-dimensional nanomaterials. ACS Nano. 9, 9451-9469 (2015)

13. Xue, Y., Zhang, Q., Wang, W., Cao, H., Yang, Q., Fu, L.: Opening two-dimensional materials for energy conversion and storage: a concept. Adv. Energy Mate. DOI: 10.1002/ aenm.2016026841602684-n/a. 
14. Tao, H., Gao, Y., Talreja, N., Guo, F., Texter, J., Yan, C., Sun, Z.: Two-dimensional nanosheets for electrocatalysis in energy generation and conversion. J. Mater. Chem. A. 5, 7257-7284 (2017)

15. Zhou, Q., Chen, Y., Zhao, G., Lin, Y., Yu, Z., Xu, X., Wang, X., Liu, H.K., Sun, W., Dou, S.X.: Active-site-enriched iron-doped nickel/cobalt hydroxide nanosheets for enhanced oxygen evolution reaction. ACS Catal. 8, 5382-5390 (2018)

16. Wang, Y., Mao, J., Meng, X., Yu, L., Deng, D., Bao, X.: Catalysis with two-dimensional materials confining single atoms: concept, design, and applications. Chem. Rev. 119, 1806-1854 (2019)

17. Chen, G., Xu, C., Huang, X., Ye, J., Gu, L., Li, G., Tang, Z., Wu, B., Yang, H., Zhao, Z., Zhou, Z., Fu, G., Zheng, N.: Interfacial electronic effects control the reaction selectivity of platinum catalysts. Nat. Mater. 15, 564 (2016)

18. Choi, C.H., Kim, M., Kwon, H.C., Cho, S.J., Yun, S., Kim, H.-T., Mayrhofer, K.J.J., Kim, H., Choi, M.: Tuning selectivity of electrochemical reactions by atomically dispersed platinum catalyst. Nat. Commun. 7, 10922 (2016)

19. Han, L., Dong, S., Wang, E.: Transition-metal (Co, Ni, and Fe)based electrocatalysts for the water oxidation reaction. Adv. Mater. 28, 9266-9291 (2016)

20. Tang, C., Wang, H.-F., Chen, X., Li, B.-Q., Hou, T.-Z., Zhang, B., Zhang, Q., Titirici, M.-M., Wei, F.: Topological defects in metal-free nanocarbon for oxygen electrocatalysis. Adv. Mater. 28, 6845-6851 (2016)

21. Strmcnik, D., Lopes, P.P., Genorio, B., Stamenkovic, V.R., Markovic, N.M.: Design principles for hydrogen evolution reaction catalyst materials. Nano Energy. 29, 29-36 (2016)

22. Seh, Z.W., Kibsgaard, J., Dickens, C.F., Chorkendorff, I., Nørskov, J.K., Jaramillo, T.F.: Combining theory and experiment in electrocatalysis: insights into materials design. Science. 355, eaad4998 (2017)

23. Novoselov, K.S., Mishchenko, A., Carvalho, A., Castro Neto, A.H.: 2D materials and van der Waals heterostructures. Science. 353, aac943 (2016)

24. Jaramillo, T.F., Jørgensen, K.P., Bonde, J., Nielsen, J.H., Horch, S., Chorkendorff, I.: Identification of active edge sites for electrochemical $\mathrm{H} 2$ evolution from MoS2 nanocatalysts. Science. 317, 100-102 (2007)

25. Zheng, Y., Jiao, Y., Zhu, Y., Li, L.H., Han, Y., Chen, Y., Du, A., Jaroniec, M., Qiao, S.Z.: Hydrogen evolution by a metal-free electrocatalyst. Nat. Commun. 5, 3783 (2014)

26. Zhu, Y.P., Guo, C., Zheng, Y., Qiao, S.Z.: Surface and Interface engineering of noble-metal-free electrocatalysts for efficient energy conversion processes. Acc. Chem. Res. (2017). https:// doi.org/10.1021/acs.accounts.6b00635

27. Zhang, L., Xia, Z.: Mechanisms of oxygen reduction reaction on nitrogen-doped graphene for fuel cells. J. Phys. Chem. C. 115, 11170-11176 (2011)

28. Deng, D., Pan, X., Yu, L., Cui, Y., Jiang, Y., Qi, J., Li, W.-X., Fu, Q., Ma, X., Xue, Q., Sun, G., Bao, X.: Toward N-doped graphene via solvothermal synthesis. Chem. Mater. 23, 1188-1193 (2011)

29. Li, Y., Zhou, Z., Shen, P., Chen, Z.: Spin gapless semiconductormetal-half-metal properties in nitrogen-doped zigzag graphene nanoribbons. ACS Nano. 3, 1952-1958 (2009)

30. Jiao, Y., Zheng, Y., Davey, K., Qiao, S.-Z.: Activity origin and catalyst design principles for electrocatalytic hydrogen evolution on heteroatom-doped graphene. Nat. Energy. 1, 16130 (2016)

31. Qu, K., Zheng, Y., Zhang, X., Davey, K., Dai, S., Qiao, S.Z.: Promotion of electrocatalytic hydrogen evolution reaction on nitrogen-doped carbon nanosheets with secondary heteroatoms. ACS Nano. 11, 7293-7300 (2017)

32. Wang, X., Wang, J., Wang, D., Dou, S., Ma, Z., Wu, J., Tao, L., Shen, A., Ouyang, C., Liu, Q., Wang, S.: One-pot synthesis of nitrogen and sulfur co-doped graphene as efficient metal-free electrocatalysts for the oxygen reduction reaction. Chem. Commun. 50, 4839-4842 (2014)

33. Huang, X., Leng, M., Xiao, W., Li, M., Ding, J., Tan, T.L., Lee, W.S.V., Xue, J.: Activating basal planes and S-terminated edges of MoS2 toward more efficient hydrogen evolution. Adv. Funct. Mater. 27, 1604943 (2017)

34. Xie, J., Zhang, J., Li, S., Grote, F., Zhang, X., Zhang, H., Wang, R., Lei, Y., Pan, B., Xie, Y.: Controllable disorder engineering in oxygen-incorporated MoS2 ultrathin nanosheets for efficient hydrogen evolution. J. Am. Chem. Soc 135, 17881-17888 (2013)

35. Zhang, J., Wang, T., Liu, P., Liu, S., Dong, R., Zhuang, X., Chen, M., Feng, X.: Engineering water dissociation sites in MoS2 nanosheets for accelerated electrocatalytic hydrogen production. Energy Environ. Sci. 9, 2789-2793 (2016)

36. Zhao, G., Wang, X., Wang, S., Rui, K., Chen, Y., Yu, H., Ma, J. Dou, S.X., Sun, W.: Heteroatom-doped MoSe2 nanosheets with enhanced hydrogen evolution kinetics for alkaline water splitting. Chem. Asian J. 14, 301-306 (2019)

37. Ma, X., Li, J., An, C., Feng, J., Chi, Y., Liu, J., Zhang, J., Sun, Y.: Ultrathin $\mathrm{Co}(\mathrm{Ni})$-doped MoS2 nanosheets as catalytic promoters enabling efficient solar hydrogen production. Nano Res. 9, 2284-2293 (2016)

38. Lau, T.H.M., Lu, X., Kulhavý, J., Wu, S., Lu, L., Wu, T.-S., Kato, R., Foord, J.S., Soo, Y.-L., Suenaga, K., Tsang, S.C.E.: Transition metal atom doping of the basal plane of MoS2 monolayer nanosheets for electrochemical hydrogen evolution. Chem. Sci. 9, 4769-4776 (2018)

39. Gao, G., Sun, Q., Du, A.: Activating catalytic inert basal plane of molybdenum disulfide to optimize hydrogen evolution activity via defect doping and strain engineering. J. Phys. Chem. C. 120, 16761-16766 (2016)

40. Zhang, L., Jia, Y., Gao, G., Yan, X., Chen, N., Chen, J., Soo, M.T., Wood, B., Yang, D., Du, A., Yao, X.: Graphene defects trap atomic ni species for hydrogen and oxygen evolution reactions. Chem. 4, 285-297 (2018)

41. Yan, X., Jia, Y., Yao, X.: Defects on carbons for electrocatalytic oxygen reduction. Soc. Rev, Chem (2018). https://doi.org/10.1039/ C7CS00690J

42. Chen, Y., Huang, S., Ji, X., Adepalli, K., Yin, K., Ling, X., Wang, X., Xue, J., Dresselhaus, M., Kong, J., Yildiz, B.: Tuning electronic structure of single layer MoS2 through defect and interface engineering. ACS Nano. 12, 2569-2579 (2018)

43. Zhang, J., Wang, Y., Cui, J., Wu, J., Li, Y., Zhu, T., Kang, H., Yang, J., Sun, J., Qin, Y., Zhang, Y., Ajayan, P.M., Wu, Y.: WaterSoluble defect-rich MoS2 ultrathin nanosheets for enhanced hydrogen evolution. J. Phys. Chem. Lett. 10, 3282-3289 (2019)

44. Li, H., Tsai, C., Koh, A.L., Cai, L., Contryman, A.W., Fragapane, A.H., Zhao, J., Han, H.S., Manoharan, H.C., Abild-Pedersen, F., Nørskov, J.K., Zheng, X.: Activating and optimizing MoS2 basal planes for hydrogen evolution through the formation of strained sulphur vacancies. Nat. Mater. 15, 48-54 (2015)

45. Ouyang, Y., Ling, C., Chen, Q., Wang, Z., Shi, L., Wang, J.: Activating inert basal planes of MoS2 for hydrogen evolution reaction through the formation of different intrinsic defects. Chem. Mater. 28, 4390-4396 (2016)

46. Tsai, C., Li, H., Park, S., Park, J., Han, H.S., Nørskov, J.K., Zheng, X., Abild-Pedersen, F.: Electrochemical generation of sulfur vacancies in the basal plane of MoS2 for hydrogen evolution. Nat. Commun. 8, 15113 (2017)

47. Li, H., Du, M., Mleczko, M.J., Koh, A.L., Nishi, Y., Pop, E., Bard, A.J., Zheng, X.: Kinetic study of hydrogen evolution reaction over strained MoS2 with sulfur vacancies using scanning electrochemical microscopy. J. Am. Chem. Soc. 138, 5123-5129 (2016)

48. Xie, J., Zhang, H., Li, S., Wang, R., Sun, X., Zhou, M., Zhou, J., Lou, X.W., Xie, Y.: Defect-rich MoS2 ultrathin nanosheets with 
additional active edge sites for enhanced electrocatalytic hydrogen evolution. Adv. Mater. 25, 5807-5813 (2013)

49. Ye, G., Gong, Y., Lin, J., Li, B., He, Y., Pantelides, S.T., Zhou, W., Vajtai, R., Ajayan, P.M.: Defects engineered monolayer MoS2 for improved hydrogen evolution reaction. Nano Lett. 16, 1097-1103 (2016)

50. Li, H., Tsai, C., Koh, A.L., Cai, L., Contryman, A.W., Fragapane, A.H., Zhao, J., Han, H.S., Manoharan, H.C., Abild-Pedersen, F., Nørskov, J.K., Zheng, X.: Activating and optimizing MoS2 basal planes for hydrogen evolution through the formation of strained sulphur vacancies. Nat. Mater. 15, 48-53 (2016)

51. Jia, Y., Zhang, L., Du, A., Gao, G., Chen, J., Yan, X., Brown, C.L., Yao, X.: Defect graphene as a trifunctional catalyst for electrochemical reactions. Adv. Mater. 28, 9532-9538 (2016)

52. Zhang, J., Wang, T., Pohl, D., Rellinghaus, B., Dong, R., Liu, S., Zhuang, X., Feng, X.: Interface engineering of MoS2/Ni3S2 heterostructures for highly enhanced electrochemical overall-watersplitting activity. Angew. Chem. Int. Edit. 55, 6702-6707 (2016)

53. Zhao, G., Lin, Y., Rui, K., Zhou, Q., Chen, Y., Dou, S.X., Sun, W.: Epitaxial growth of $\mathrm{Ni}(\mathrm{OH}) 2$ nanoclusters on MoS2 nanosheets for enhanced alkaline hydrogen evolution reaction. Nanoscale. 10, 19074-19081 (2018)

54. Chen, Y., Wang, X., Lao, M., Rui, K., Zheng, X., Yu, H., Ma, J., Dou, S.X., Sun, W.: Electrocatalytically inactive $\mathrm{SnS} 2$ promotes water adsorption/dissociation on molybdenum dichalcogenides for accelerated alkaline hydrogen evolution. Nano Energy. 64, 103918 (2019)

55. Zhou, Q., Zhao, G., Rui, K., Chen, Y., Xu, X., Dou, S.X., Sun, $\mathrm{W}$.: Engineering additional edge sites on molybdenum dichalcogenides toward accelerated alkaline hydrogen evolution kinetics. Nanoscale. 11, 717-724 (2019)

56. Duan, J., Chen, S., Jaroniec, M., Qiao, S.Z.: Porous C3N4 Nanolayers@N-graphene films as catalyst electrodes for highly efficient hydrogen evolution. ACS Nano. 9, 931-940 (2015)

57. Lu, Z., Wang, B., Hu, Y., Liu, W., Zhao, Y., Yang, R., Li, Z., Luo, J., Chi, B., Jiang, Z., Li, M., Mu, S., Liao, S., Zhang, J., Sun, X.: An isolated zinc-cobalt atomic pair for highly active and durable oxygen reduction. Angew. Chem. Int. Ed. 58, 2622-2626 (2019)

58. Qi, K., Cui, X., Gu, L., Yu, S., Fan, X., Luo, M., Xu, S., Li, N., Zheng, L., Zhang, Q., Ma, J., Gong, Y., Lv, F., Wang, K., Huang, H., Zhang, W., Guo, S., Zheng, W., Liu, P.: Single-atom cobalt array bound to distorted 1T MoS2 with ensemble effect for hydrogen evolution catalysis. Nat. Commun. 10, 5231 (2019)

59. Zhang, J., Zhao, Y., Guo, X., Chen, C., Dong, C.-L., Liu, R.-S., Han, C.-P., Li, Y., Gogotsi, Y., Wang, G.: Single platinum atoms immobilized on an MXene as an efficient catalyst for the hydrogen evolution reaction. Nat. Catal. 1, 985-992 (2018)

60. Ye, S., Luo, F., Zhang, Q., Zhang, P., Xu, T., Wang, Q., He, D., Guo, L., Zhang, Y., He, C., Ouyang, X., Gu, M., Liu, J., Sun, X.: Highly stable single Pt atomic sites anchored on aniline-stacked graphene for hydrogen evolution reaction. Energy Environ. Sci. 12, 1000-1007 (2019)

61. Qiu, H.-J., Ito, Y., Cong, W., Tan, Y., Liu, P., Hirata, A., Fujita, T., Tang, Z., Chen, M.: Nanoporous graphene with single-atom nickel dopants: an efficient and stable catalyst for electrochemical hydrogen production. Angew. Chem. Int. Edit. 54, 14031-14035 (2015)

62. Fei, H., Dong, J., Arellano-Jiménez, M.J., Ye, G., Dong Kim, N., Samuel, E.L.G., Peng, Z., Zhu, Z., Qin, F., Bao, J., Yacaman, M.J., Ajayan, P.M., Chen, D., Tour, J.M.: Atomic cobalt on nitrogendoped graphene for hydrogen generation. Nat. Commun. 6, 8668 (2015)

63. Voiry, D., Salehi, M., Silva, R., Fujita, T., Chen, M., Asefa, T., Shenoy, V.B., Eda, G., Chhowalla, M.: Conducting MoS2 nanosheets as catalysts for hydrogen evolution reaction. Nano Lett. 13, 6222-6227 (2013)

64. Lukowski, M.A., Daniel, A.S., English, C.R., Meng, F., Forticaux, A., Hamers, R.J., Jin, S.: Highly active hydrogen evolution catalysis from metallic WS2 nanosheets. Energy Environ. Sci. 7, 2608-2613 (2014)

65. Geng, X., Sun, W., Wu, W., Chen, B., Al-Hilo, A., Benamara, M., Zhu, H., Watanabe, F., Cui, J., Chen, T.-P.: Pure and stable metallic phase molybdenum disulfide nanosheets for hydrogen evolution reaction. Nat. Commun. 7, 10672 (2016)

66. Zhang, X., Zhang, Y.-Y., Zhang, Y., Jiang, W.-J., Zhang, Q.-H., Yang, Y.-G., Gu, L., Hu, J.-S., Wan, L.-J.: Phase-controlled synthesis of $1 \mathrm{~T}-\mathrm{MoSe} / \mathrm{NiSe}$ heterostructure nanowire arrays via electronic injection for synergistically enhanced hydrogen evolution. Small Methods. 3, 1800317 (2018)

67. Voiry, D., Yamaguchi, H., Li, J., Silva, R., Alves, D.C.B., Fujita, T., Chen, M., Asefa, T., Shenoy, V.B., Eda, G., Chhowalla, M.: Enhanced catalytic activity in strained chemically exfoliated WS2 nanosheets for hydrogen evolution. Nat. Mater. 12, 850-855 (2013)

68. Yin, Y., Zhang, Y., Gao, T., Yao, T., Zhang, X., Han, J., Wang, X., Zhang, Z., Xu, P., Zhang, P., Cao, X., Song, B., Jin, S.: Synergistic phase and disorder engineering in $1 \mathrm{~T}-\mathrm{MoSe} 2$ nanosheets for enhanced hydrogen-evolution reaction. Adv. Mater. 29, 1700311 (2017)

69. Zang, X., Chen, W., Zou, X., Hohman, J.N., Yang, L., Li, B., Wei, M., Zhu, C., Liang, J., Sanghadasa, M., Gu, J., Lin, L.: Self-assembly of large-area 2D polycrystalline transition metal carbides for hydrogen electrocatalysis. Adv. Mater. 30, 1805188 (2018)

70. Zhou, M., Zhang, Z., Huang, K., Shi, Z., Xie, R., Yang, W.: Colloidal preparation and electrocatalytic hydrogen production of MoS2 and WS2 nanosheets with controllable lateral sizes and layer numbers. Nanoscale. 8, 15262-15272 (2016)

71. Guo, Y., Xu, K., Wu, C., Zhao, J., Xie, Y.: Surface chemicalmodification for engineering the intrinsic physical properties of inorganic two-dimensional nanomaterials. Chem. Soc. Rev. 44, 637-646 (2015)

72. Deng, D., Novoselov, K.S., Fu, Q., Zheng, N., Tian, Z., Bao, X. Catalysis with two-dimensional materials and their heterostructures. Nat. Nanotech. 11, 218-230 (2016)

73. Wang, T., Liu, L., Zhu, Z., Papakonstantinou, P., Hu, J., Liu, H., Li, M.: Enhanced electrocatalytic activity for hydrogen evolution reaction from self-assembled monodispersed molybdenum sulfide nanoparticles on an Au electrode. Energy Environ. Sci. 6, 625-633 (2013)

74. Yu, Y., Huang, S.-Y., Li, Y., Steinmann, S.N., Yang, W., Cao, L.: Layer-Dependent Electrocatalysis of MoS2 for Hydrogen Evolution. Nano Lett. 14, 553-558 (2014)

75. Hong Ng, V.M., Huang, H., Zhou, K., Lee, P.S., Que, W., Xu, J.Z., Kong, L.B.: Recent progress in layered transition metal carbides and/or nitrides (MXenes) and their composites: synthesis and applications. J. Mater. Chem. A. 5, 3039-3068 (2017)

76. Anasori, B., Lukatskaya, M.R., Gogotsi, Y.: 2D metal carbides and nitrides (MXenes) for energy storage. Nat. Rev. Mater. 2, 16098 (2017)

Publisher's Note Springer Nature remains neutral with regard to jurisdictional claims in published maps and institutional affiliations. 